\title{
Cost-effectiveness of child caries management: a randomised controlled trial (FiCTION trial)
}

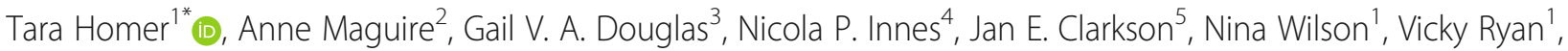
Elaine McColl ${ }^{1}$, Mark Robertson ${ }^{4}$ and Luke Vale ${ }^{1}$

\begin{abstract}
Background: A three-arm parallel group, randomised controlled trial set in general dental practices in England, Scotland, and Wales was undertaken to evaluate three strategies to manage dental caries in primary teeth. Children, with at least one primary molar with caries into dentine, were randomised to receive Conventional with best practice prevention $(C+P)$, Biological with best practice prevention $(B+P)$, or best practice Prevention Alone (PA).

Methods: Data on costs were collected via case report forms completed by clinical staff at every visit. The coprimary outcomes were incidence of, and number of episodes of, dental pain and/or infection avoided. The three strategies were ranked in order of mean cost and a more costly strategy was compared with a less costly strategy in terms of incremental cost-effectiveness. Costs and outcomes were discounted at 3.5\%.

Results: A total of 1144 children were randomised with data on 1058 children $(C+P n=352, B+P n=352$, $P A n=$ $354)$ used in the analysis. On average, it costs $£ 230$ to manage dental caries in primary teeth over a period of up to 36 months. Managing children in PA was, on average, $£ 19$ (97.5\% Cl: $-£ 18$ to $£ 55)$ less costly than managing those in $\mathrm{B}+\mathrm{P}$. In terms of effectiveness, on average, there were fewer incidences of, $(-0.06 ; 97.5 \% \mathrm{Cl}$ : -0.14 to 0.02$)$ and fewer episodes of dental pain and/or infection $(-0.14 ; 97.5 \% \mathrm{Cl}$ : -0.29 to 0.71$)$ in B + P compared to PA. C + P was unlikely to be considered cost-effective, as it was more costly and less effective than $\mathrm{B}+\mathrm{P}$.

Conclusions: The mean cost of a child avoiding any dental pain and/or infection (incidence) was $£ 330$ and the mean cost per episode of dental pain and/or infection avoided was $£ 130$. At these thresholds $B+P$ has the highest probability of being considered cost-effective. Over the willingness to pay thresholds considered, the probability of $\mathrm{B}+\mathrm{P}$ being considered cost-effective never exceeded $75 \%$.

Trial registration: The trial was prospectively registered with the ISRCTN (reference number ISRCTN77044005) on the 26th January 2009 and East of Scotland Research Ethics Committee provided ethical approved (REC reference: 12/ES/0047).
\end{abstract}

Keywords: Economic evaluation, Caries, Caries treatment, Clinical studies/trials, Pediatric dentistry, Dental public health

\footnotetext{
* Correspondence: tara.homer@newcastle.ac.uk

${ }^{1}$ Population Health Sciences Institute, Newcastle University, Newcastle upon

Tyne NE2 4AX, UK

Full list of author information is available at the end of the article
}

(c) The Author(s). 2020 Open Access This article is distributed under the terms of the Creative Commons Attribution 4.0 International License (http://creativecommons.org/licenses/by/4.0/), which permits unrestricted use, distribution, and reproduction in any medium, provided you give appropriate credit to the original author(s) and the source, provide a link to the Creative Commons license, and indicate if changes were made. The Creative Commons Public Domain Dedication waiver (http://creativecommons.org/publicdomain/zero/1.0/) applies to the data made available in this article, unless otherwise stated. 


\section{Background}

Dental caries has a large health and economic impact for the United Kingdom (UK) as it is the most common disease in children [1-4]. Treating oral disease is expensive, costing NHS England $£ 3.4$ billion annually [5].

In the UK there is uncertainty surrounding the best strategy to manage caries in primary teeth, especially in primary care. There is debate about the clinical- and cost-effectiveness of conventional restorations (removing a carious lesion with a drill and placement of a restoration) compared to minimallyinvasive biologically-orientated strategies (sealing-in a carious lesion with an adhesive restoration or preformed metal crown rather than removing it), or prevention-focused strategies [6-9].

Cost-effectiveness analysis allows treatment comparisons in terms of both costs and effects [10]. Recent cost-effectiveness analyses of managing dental caries found the Hall Technique (HT), a method for managing carious lesions by sealing-in, to be costeffective compared to conventional restorations [11] and compared to both conventional restorations and a Non-Restorative Cavity Control approach [12] However, these studies followed outcomes on single teeth and have focused on one type of biological approach (i.e. HT).

A large trial, FiCTION (Filling Children's Teeth: Indicated or Not?), was undertaken to measure the costs and effects, in terms of dental pain and/or infection, of three strategies to manage dental caries in the primary teeth of young children with dentine caries in the UK [13]. The strategies evaluated were Conventional restorations with best practice prevention $(\mathrm{C}+\mathrm{P})$, Biological management of carious lesions with best practice prevention $(B+P)$, and best practice Prevention Alone (PA). The $\mathrm{C}+\mathrm{P}$ strategy involved the complete mechanical removal of carious tooth tissue using local anesthesia and a drill followed by placement of a restoration alongside best practice preventive therapy and has been considered standard practice in the management of dental caries $[14,15]$. The $\mathrm{B}+\mathrm{P}$ strategy involved sealing-in carious lesions using a variety of techniques including adhesive restorative materials or preformed metal crowns placed using the HT along with preventive therapy; Schwendicke et al.'s (2018, 2019) analyses focused on the HT component of $\mathrm{B}+\mathrm{P}[11,12]$. PA involved avoiding restorative intervention and using four components of preventive management; toothbrushing (with toothpaste of at least 1000ppmF concentration), dietary advice, fluoride varnish application, and fissure sealants to prevent further carious lesions.
The trial methodology and clinical outcomes are presented elsewhere [13, 16, 17]. In brief, this multicenter, three-arm, parallel group, patient-randomised controlled trial set in general dental practices in England, Scotland, and Wales was undertaken to evaluate three strategies to manage caries in the primary teeth of children aged 3 to 7 years with at least one primary molar tooth with caries lesions extending into dentine. The original planned follow-up was 3 years but due to an extension in the recruitment period this was revised to an average target follow-up of 35.5 months (a minimum of 23 months and a maximum of 36 months).

\section{Methods}

Reporting for this study follows the Consolidated Health Economic Evaluation Reporting Standards (CHEERS) [18]. The trial was registered with the ISRCTN (reference number ISRCTN77044005) and East of Scotland Research Ethics Committee provided ethical approved (REC reference: 12/ES/0047).

\section{Data analyses}

The economic evaluation was undertaken from the perspective of the healthcare provider in the UK, the National Health Service (NHS).

\section{Estimation of costs}

Time/materials-based costing was used to estimate the costs at every visit to manage dental caries in primary teeth. These costs depended on the quantity of dental care resources used for each child during their time in the trial (up to 36 months postrandomisation). Resource use data, to inform the cost analysis, were collected via case report forms (CRFs) completed by the clinician at every visit. Costs were categorised as staffing, preventive treatments, operative treatments (restoration materials), other associated items (e.g. radiographs), referrals, and prescriptions. Capital costs were excluded as all three strategies were provided as part of current care; therefore, these costs would have been incurred regardless of which strategy was implemented. Unit costs, based on the materials required for each treatment, were multiplied by the number of resources used. Unit costs are detailed in Additional file 1 and briefly described below. All costs are in 2018 pounds Sterling.

The length of time for each visit, based on the start and end time recorded in the CRF, was used to estimate dental personnel costs. Time spent providing prevention was subtracted from total visit time to take into account that the same personnel may not 
provide preventive and operative treatments. We assumed a dental nurse would be present for the full duration of each visit.

Preventive care was integral to all three arms and was expected to be provided regardless of randomised allocation. Preventive treatment costs were the resources used for fluoride applications and fissure sealants placed on first permanent molars.

Operative treatments were included in two arms; $\mathrm{C}+\mathrm{P}$ (e.g. local anesthetic, carious tissue removal, and restoration) and $\mathrm{B}+\mathrm{P}$ (e.g. partial/no carious tissue removal and restoration), but some treatments were included in all three arms (e.g. extractions under local anesthetic and pulp therapy). Information on the number of surfaces treated was also collected since treatment of more than one tooth surface could incur additional costs (e.g. additional restorative material). The cost of resources used at every visit were also included, regardless of treatment. Other treatment costs included radiographs and inhalation sedation.

A patient referral was reported if a child was referred to a dental hospital/clinic for a consultation and/or operative treatment. The costs associated with referrals were categorised A-F (see Additional file 2) depending on treatment provided, where it took place, who provided it, and the number of visits required.

\section{Estimation of effects}

The original primary outcome, incidence of dental pain and/or infection was modified during the trial to include a co-primary outcome, number of episodes of dental pain and/or infection. Number of episodes was included as it was considered more clinically relevant and statistically more sensitive to analyse the frequency of dental pain and/or infection experienced by a child.
Incidence is defined as the proportion of children with at least one episode of dental pain and/or infection during their time in the study. Episodes were defined on a tooth-by-tooth basis based on the frequency of dental pain and/or infection reported during the child's follow-up. However, if multiple teeth had dental pain and/or infection at the same visit, this was counted as one episode or if the same tooth had dental pain and/or infection at consecutive visits, this was counted as one episode regardless of the time between visits [13]. Data on dental pain due to dental caries and clinically diagnosed infection were collected on the CRF at every visit. It was assumed that those who did not have regular appointments did not need further treatment and/or did not experience dental pain and/or infection.

\section{Cost-effectiveness analysis}

The economic analysis was conducted on the basis of intention-to-treat (ITT). Children were included in the ITT analysis if they had at least one CRF and therefore at least one clinical assessment of the primary outcome. The economic analysis compared the three strategies in terms of mean costs and effects over the follow-up period. Both costs and effects were discounted at the recommended rate of $3.5 \%$ [19]. Effects were discounted, based on when the incidence or episode of dental pain and/or infection began. To enable the estimation of budget impact [20] the average total costs by cost category presented in Table 1 were not discounted.

For the incremental analysis, the strategies were ranked in terms of increasing mean cost and a more costly strategy was compared with a less costly strategy in terms of incremental cost-effectiveness. A treatment was considered to be dominated if it was

Table 1 Average total cost $(£)$ per child by strategy ${ }^{a}$

\begin{tabular}{|c|c|c|c|}
\hline & \multicolumn{3}{|c|}{ Total cost per child (£) } \\
\hline & $C+P$ & $B+P$ & $\mathrm{PA}$ \\
\hline Resource & Mean [SD] & Mean [SD] & Mean [SD] \\
\hline Staff costs & $18.78[6.07]$ & $18.28[6.27]$ & $17.36[5.95]$ \\
\hline Prevention costs & $0.66[0.76]$ & $0.78[0.88]$ & $0.81[0.88]$ \\
\hline Operative treatment costs & $8.18[6.72]$ & $7.84[5.96]$ & $4.09[4.05]$ \\
\hline Other treatments costs & $0.66[2.56]$ & $0.47[1.84]$ & $0.52[1.90]$ \\
\hline Referral costs & $5.22[23.35]$ & $4.96[23.65]$ & $10.23[43.81]$ \\
\hline Prescription costs & $0.07[0.29]$ & $0.04[0.14]$ & $0.08[0.32]$ \\
\hline Total practice level treatment cost (exc. referrals) per child per visit & $28.36[11.08]$ & $27.40[10.81]$ & $22.86[8.11]$ \\
\hline Total treatment cost per child & $250.48(221.70)$ & $231.27(214.47)$ & $211.32(257.28)$ \\
\hline
\end{tabular}

${ }^{a}$ costs are not discounted in this table but presented in the common price year to allow for budget impact 
more costly and less effective than its comparator [10]. If a treatment was not dominated, an incremental cost-effectiveness ratio (ICER) was estimated. The ICER is the difference in mean costs divided by the difference in mean effects and gives an estimate of the mean cost per additional unit of effect [10].

STATA software was used for all analyses [21]. Regressions on costs and effects were run simultaneously using seemingly unrelated regression (SUR) [22]. SUR permits the simultaneous estimation of costs and effects, calculated at an individual level, while accounting for unobserved individual characteristics that could affect both costs and effects and lead to potential correlation between these two dependent variables [23]. In addition, the SUR controlled for additional covariates (age, time in study, and practice variation) that may affect costs, effects, or both.

A stochastic sensitivity analysis, using the bootstrapping technique [24], explored the impact of the statistical imprecision surrounding estimates of costs, effects, and cost-effectiveness. The bootstrapped results from the incremental analysis were used to estimate net benefits (NB). The NB statistic is given by:

$$
\mathrm{NB}=(\lambda \times \Delta \mathrm{e})-\Delta \mathrm{c}
$$

where $\lambda$ is the willingness to pay threshold, $\Delta$ is the difference between a strategy and its comparator (i.e. least costly strategy), e are the mean effects, and $\mathrm{c}$ are the mean costs [10]. A strategy is considered to be costeffective if $\mathrm{NB}>0$ or, when more than two strategies are compared, a strategy which has the highest NB at a given threshold value for society's willingness to pay for a unit of oral health benefit. As there is no nationally or internationally agreed willingness to pay threshold to avoid dental pain and/or infection an arbitrary threshold of $£ 1000$, used by O'Neill et al. (2017), was adopted for this analysis [25]. A costeffectiveness frontier [26] was generated to illustrate uncertainty by showing which strategy was likely to have the highest NB over a range of different willingness to pay values.

\section{Results}

A total of 1144 children were randomised and data on 1058 children were used in the economic analysis $(n=86$ children did not have any clinical assessment of the primary outcome and were not included in the ITT analysis). The children included in the economic analysis were evenly distributed across the three arms in terms of numbers randomised and baseline characteristics; 352 randomised to $\mathrm{B}+\mathrm{P}, 352$ to $\mathrm{C}+\mathrm{P}$, and 354 to $\mathrm{PA}$. On average, children were
6 years old [sd: 1.3] when recruited and there was an even split between females (51\%) and males (49\%). The CONSORT (Consolidated Standards of Reporting Trial) flow diagram is provided in Additional file 6 but additional baseline characteristics, and clinical findings are presented elsewhere [13]. The median follow-up was 33.8 months (IQR 23.8, 36.7).

The percentage of missing data for the economic analysis was low $(<5 \%)$. There were 7713 visits recorded across the three arms. On average, children had seven visits during their time in the trial, each lasting $21 \mathrm{~min}$. All three strategies were similar in terms of average number of visits (mean visits [sd]: C + P 7.7 [4.2], B + P 7.4 [4.1], and PA 6.8 [3.7]) and duration of visits (mean minutes [sd]: C + P 21.8 [6.9], B + P 21.2 [7.2], and PA 20.1 [6.7]).

The number of visits at which preventive treatment was provided was similar across the three arms with slightly more prevention provided in the PA arm $(\mathrm{C}+\mathrm{P} 79 \%, \mathrm{~B}+\mathrm{P} 79 \%$, and $\mathrm{PA} 85 \%$ of visits). The three strategies differed in the frequency of operative treatments provided, with less than $20 \%$ of all PA visits involving operative treatment compared to over $40 \%$ of $\mathrm{B}+\mathrm{P}$ and $\mathrm{C}+\mathrm{P}$ visits. The type of operative treatment provided also differed, as would be expected given the nature of the different strategies.

A total of 96 children $(\mathrm{C}+\mathrm{P} n=31, \mathrm{~B}+\mathrm{P} n=31$, and PA $n=34$ children) were referred on 107 occasions for additional consultations and/or further treatment $(\mathrm{C}+\mathrm{P} n=32, \mathrm{~B}+\mathrm{P} n=36$, and $\mathrm{PA} n=39$ referrals) resulting in 52 general anesthetics (GA) being undertaken $(\mathrm{C}+\mathrm{P} n=15, \mathrm{~B}+\mathrm{P} n=12, \mathrm{PA} n=25$ referrals with GA). Four children did not attend their referral appointment $(n=1 \mathrm{C}+\mathrm{P}, n=3 \mathrm{PA})$.

Table 1 summarizes the average cost per child per visit for the three strategies.

On average, it cost $£ 230$ to manage dental caries in a young child with at least one primary tooth with a dentinal carious lesion over a period of up to 36 months. On average, $\mathrm{C}+\mathrm{P}$ was the most costly and PA was the least costly strategy. Staff time, operative treatments, and patient referrals were the main cost drivers. As expected, $\mathrm{C}+\mathrm{P}$ and $\mathrm{B}+\mathrm{P}$ incurred more operative treatment costs compared to $\mathrm{PA}$.

As PA was, on average, the least costly strategy we compared this to $\mathrm{B}+\mathrm{P}$, the next costly strategy, and lastly $\mathrm{C}+\mathrm{P}$ was included in the comparison. In terms of effectiveness, there was no evidence of a difference in incidence, or in episodes, of dental pain and/or infection between the three strategies. Table 2 summaries the results of the incremental analysis.

$\mathrm{B}+\mathrm{P}$ was, on average, more costly but more effective, in terms of both incidence of, and episodes of, dental pain and/or infection avoided, compared to 
Table 2 Cost-effectiveness analysis for the comparison of PA vs B $+\mathrm{P}$ vs $C+\mathrm{P}^{\mathrm{a}}$

\begin{tabular}{|c|c|c|c|c|c|}
\hline Investigation strategy & Cost $[£][97.5 \% \mathrm{Cl}]^{\mathrm{b}}$ & $\begin{array}{l}\text { Incremental cost }[£] \\
{[97.5 \% \mathrm{Cl}]^{\mathrm{b} c}}\end{array}$ & Incidence $[97.5 \% \mathrm{Cl}]^{\mathrm{b}}$ & $\begin{array}{l}\text { Incremental incidence } \\
{[97.5 \% \mathrm{Cl}]^{\mathrm{b} c}}\end{array}$ & $\mathrm{ICER}^{\mathrm{C}}[£]$ \\
\hline \multicolumn{6}{|c|}{ Incremental cost per incidence of dental pain and/or infection avoided } \\
\hline PA $(n=354)$ & 206 [176 to 237] & & $0.44[0.39$ to 0.50$]$ & & \\
\hline $\mathrm{B}+\mathrm{P}(n=352)$ & $226[201$ to 252$]$ & $19[-18$ to 55$]$ & $0.39[0.33$ to 0.45$]$ & $-0.058[-0.14$ to 0.02$]$ & 328 \\
\hline$C+P(n=352)$ & $245[219$ to 271$]$ & & $0.41[0.35$ to 0.47$]$ & & Dominated by $B+P$ \\
\hline \multicolumn{6}{|c|}{ Incremental cost per episode of dental pain and/or infection avoided } \\
\hline Investigation strategy & $\begin{array}{l}\text { Cost }[£] \\
{[97.5 \% \mathrm{Cl}]^{\mathrm{b}}}\end{array}$ & $\begin{array}{l}\text { Incremental cost }[£] \\
{[97.5 \% \mathrm{Cl}]^{\mathrm{b}} \mathrm{c}}\end{array}$ & $\begin{array}{l}\text { Episodes } \\
{[97.5 \% \mathrm{Cl}]^{\mathrm{b}}}\end{array}$ & $\begin{array}{l}\text { Incremental episodes } \\
{[97.5 \% \mathrm{Cl}]^{\mathrm{b}} \mathrm{c}}\end{array}$ & $\operatorname{ICER}^{\mathrm{C}}[£]$ \\
\hline PA $(n=354)$ & $206[176$ to 237$]$ & & $0.70[0.58$ to 0.82$]$ & & \\
\hline$B+P(\underline{n}=352)$ & $226[201$ to 252$]$ & $19[-18$ to 55$]$ & $0.56[0.46$ to 0.67$]$ & $-0.143[-0.29$ to 0.01$]$ & 133 \\
\hline$C+P(n=352)$ & 245 [219 to 271$]$ & & 0.60 [0.49 to 0.71$]$ & & Dominated by $B+P$ \\
\hline
\end{tabular}

a costs and effects are discounted at $3.5 \%{ }^{b}{ }^{b} 97.5 \% \mathrm{Cl}$ was used as it adjusts for multiple comparisons and should be interpreted as if it were a $95 \% \mathrm{Cl}^{\mathrm{c}}{ }^{\mathrm{estimated}}$ based on adjusted analysis ( $n=1057 ; n=1$ child missing information on age); ${ }^{\mathrm{d}}$ ICER $=$ incremental cost-effectiveness ratio

PA. At a willingness to pay threshold of $£ 330$ we would consider $\mathrm{B}+\mathrm{P}$ cost-effective to avoid an incidence and $£ 130$ to avoid an episode of dental pain and/or infection compared to PA. As $\mathrm{C}+\mathrm{P}$ was, on average, more costly and less effective than $\mathrm{B}+\mathrm{P}$, in terms of both incidence of, and episodes of, dental pain and/or infection, it was dominated by $\mathrm{B}+\mathrm{P}$.

Figure 1 illustrates uncertainty surrounding the point estimates in Table 2. The figure presents the strategy with the highest probability of being considered cost-effective at each willingness to pay threshold to avoid an incidence of dental pain and/or infection.

Figure 1 illustrates that PA would have the highest probability $(87 \%)$ of being considered cost-effective if a decision were to be based on cost alone. However, as the willingness to pay threshold increases, the probability of $\mathrm{B}+\mathrm{P}$ being considered cost-effective increases, but it never exceeds $65 \%$. $\mathrm{C}+\mathrm{P}$ would not be considered cost-effective compared to $\mathrm{PA}$ and $\mathrm{B}+\mathrm{P}$ in this analysis.

Figure 2 illustrates the strategy with the highest probability of being considered cost-effective at the different willingness to pay thresholds to avoid an episode of dental pain and/or infection.

In terms of episodes of dental pain and/or infection, the conclusions are similar except that $\mathrm{B}+\mathrm{P}$ would be considered cost-effective at a lower willingness to pay threshold. The probability of $\mathrm{B}+\mathrm{P}$ being considered cost-effective never exceeds $75 \%$.

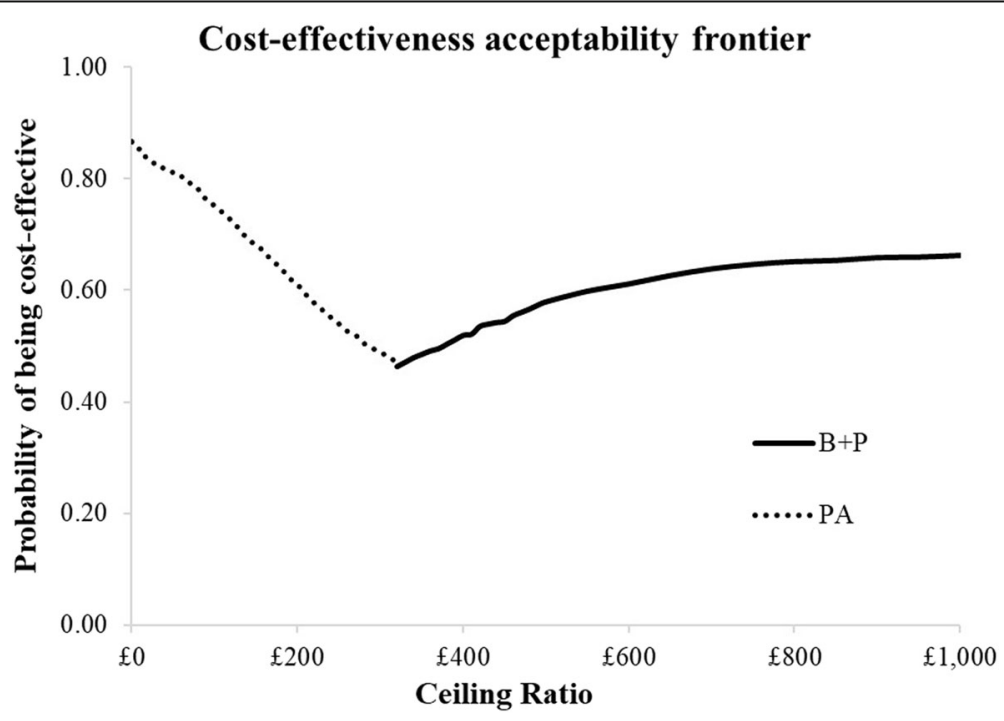

Fig. 1 Probability of being cost-effective to avoid an incidence of dental pain and/or infection 


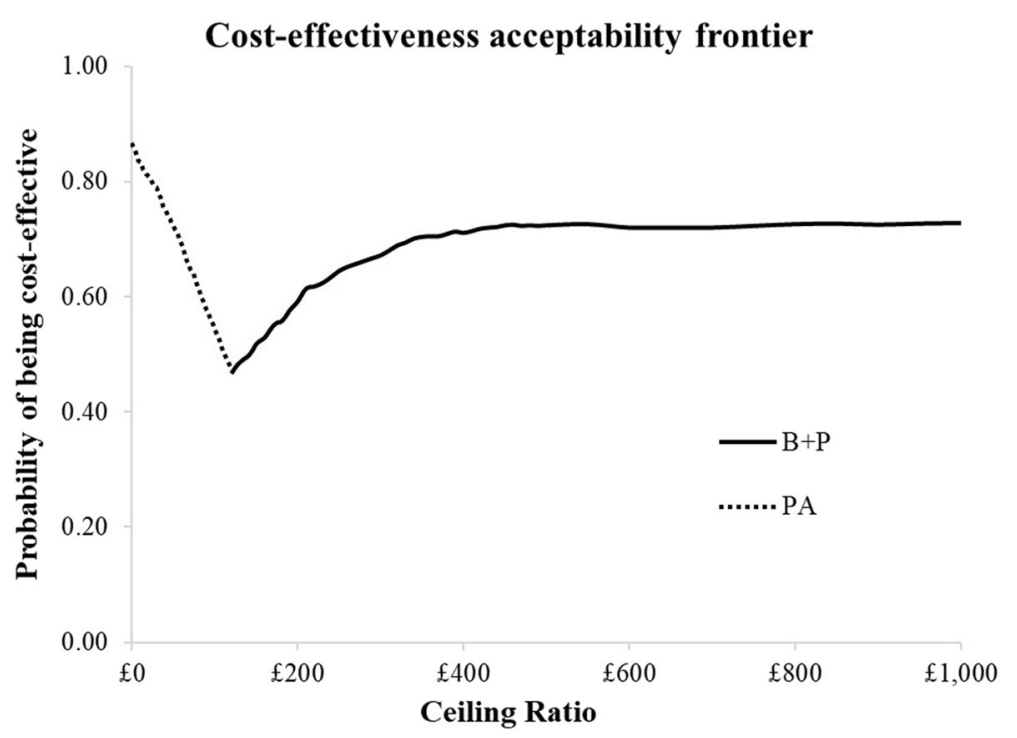

Fig. 2 Probability of being cost-effective to avoid an episode of dental pain and/or infection

\section{Discussion}

On average, it costs $£ 230$ to manage dental caries in primary teeth in a child with at least one tooth with a dentinal caries lesion over a period of up to 36 months. The main cost drivers were staff time, operative treatments, and patient referrals. On average, PA incurred a higher referral cost because that arm had more referrals and more referrals requiring $\mathrm{GA}$, an important consideration in view of the morbidity associated with GA use [27-29].

Although in terms of cost-effectiveness PA was, on average, the least costly treatment, it was also the least effective for both incidence of, and episodes of, dental pain and/or infection. There was an $87 \%$ probability that PA would be considered cost-effective as the least costly option but $\mathrm{B}+\mathrm{P}$ and $\mathrm{C}+\mathrm{P}$ would, on average, provide more oral health benefits, albeit at a higher cost. If society was willing to pay $£ 330$ to avoid one additional child experiencing dental pain and/or infection, B + P would have the highest probability (47\%) of being considered cost-effective compared to PA (46\%) and C+P (7\%). Similarly, when society is willing to pay $£ 130$ or more to avoid an episode of dental pain and/or infection, $\mathrm{B}+\mathrm{P}$ would have the highest probability $(49 \%)$ of being considered cost-effective compared to PA (45\%) and C + P (6\%).

Vermaire et al. (2014) and Samnaliev et al. (2015) came to similar conclusions in their analyses, in that treatments aimed at caries prevention increased the cost of providing treatment and that the opportunity cost of these treatments is dependent on the payers' willingness-to-pay [30, 31]. Our results differ from other studies in which the HT, which was a component of our $\mathrm{B}+\mathrm{P}$ intervention, was reported to be more effective and less costly $[11,12]$ when compared to conventional and preventive based strategies. However, both of these studies by Schwendicke et al. $(2018,2019)$ were based on treating a single tooth, or two contralateral teeth per child whereas in our study the whole child/mouth (up to 20 primary teeth per child) could be treated, a situation more representative of real life treatment provision [11, 12]. Our study also had considerably more data available to inform our analysis $(n=1058$ children, $n=2721$ teeth; compared with $n=142$ children and teeth in Schwendicke et al. 2018; and $n=91$ children, $n=182$ teeth in Schwendicke et al. 2019) [11, 12]. The costs estimated in the two Schwendicke et al. $(2018,2019)$ studies were based on current charges to the health system $[11,12]$. In the present analysis we based our costs on a very detailed costing exercise, however, when we used current charges to the NHS in a sensitivity analysis we still reached the same conclusion (see Additional files 3, 4, and 5). Schwendicke et al. (2019) found a negligible difference in total treatment costs between HT and conventional treatment and this difference only became clinically and statistically significant when patient costs were considered [11]. Parental time and travel costs to attend appointments were not considered in our analysis. Inclusion of such costs are unlikely to change our conclusions as the average number of visits and length of visits were similar across the arms. Costs incurred by the parent and child due to toothache, such as time off work, 
childcare, and time off school, were considered in a sensitivity analysis but did not change our overall conclusions. In terms of oral health effects, direct comparisons could not be made with previous studies $[11,12]$ which considered the pain associated with dental caries together with endodontic treatment and extractions. The main differences in our study are, firstly, that $\mathrm{B}+\mathrm{P}$ encompassed a number of minimally-invasive restorations of which the HT was only one and secondly, treatment was at the participant level (including all primary teeth) and not at the single tooth level.

This economic analysis had a number of strengths and limitations. The main strength was that the analysis was pre-planned and the data used were collected as part of the trial. There were few missing data and all available data were included in the analysis despite the varying follow-up. A limitation of the analysis was that SUR model may not have been an appropriate fit for the co-primary outcomes. However, a trade-off was made between fitting the most appropriate model and applying one that allows for the correlation of costs and outcomes, which the SUR approach does. Finally, capital costs were excluded from the analysis; this omission reduced the total cost of each arm equally hence the incremental costs, ICER, and our overall conclusions remain unchanged.

In practical terms, we do not know society's willingness to pay threshold to avoid dental pain and/or infection in a primary tooth. A judgement is required as to what value the NHS places on avoiding dental pain and/or infection. Recent research conducted by Lord et al. (2015) estimated the willingness to pay to avoid dental caries with pain in a primary tooth [32]. They estimated this to be $£ 153$ (95\% CI: $£ 93$ to $£ 213$ - inflated to 2017) [33]. If we adopted this as the willingness to pay threshold the PA arm would have a $68 \%$ probability of being considered cost-effective compared to B + P (29\%) and C + P (3\%) in terms of an incidence of dental pain and/or infection avoided. A willingness to pay threshold to avoid an episode of dental pain and/or infection also needs to be determined but based on the Lord et al. (2015) threshold, $\mathrm{B}+\mathrm{P}$ would have the highest probability $(53 \%)$ of being considered cost-effective compared to PA (40\%) and $\mathrm{C}+\mathrm{P}(7 \%)$. Further research is needed to identify the most appropriate threshold to assess our results.

\section{Conclusions}

To conclude, on average, PA is the least costly, despite having more referrals requiring GA, but the least effective strategy for managing dental caries in primary teeth. $\mathrm{B}+\mathrm{P}$ has the potential to provide more oral health benefits to children with dentinal carious lesion in at least one primary molar tooth, however this comes at an additional cost. Over the willingness to pay values considered, the probability of $\mathrm{B}+\mathrm{P}$ being considered cost-effective was approximately no higher than $65 \%$ to avoid an incidence of dental pain and/or infection and no higher than $75 \%$ to avoid an episode of dental pain and/or infection. It is unlikely that $\mathrm{C}+\mathrm{P}$ would be considered cost-effective.

\section{Supplementary information}

Supplementary information accompanies this paper at https://doi.org/10. 1186/s12903-020-1020-1.

Additional file 1. "Unit costs" is a table summarising the unit costs used in the analysis.

Additional file 2. "Groupings for referrals" is a table summarising the different groupings used to categorise patient referrals.

Additional file $\mathbf{3}$ "Cost-effectiveness analysis for the comparison of PA vs B+P vs C+P arms based on fee-for-service costs in Scotland only $(n=$ $287)^{\prime \prime}$ is a the results of a sensitivity analysis which estimates costs based on charges to the NHS, based on the Scottish reimbursement rates (feefor-service).

Additional file 4. "Cost-effectiveness analysis for the comparison of PA vs $B+P$ vs $C+P$ arms based on units of dental activity in England and Wales only costs $(n=771)^{\prime \prime}$ is a the results of a sensitivity analysis which estimates costs based on charges to the NHS, based on the English reimbursement rates (Units of Dental Activity).

Additional file 5. "Cost-effectiveness analysis for the comparison of PA vs B+P vs C+P based on units of dental activity (England and Wales) and fee-for service (Scotland) costs $(n=1058)^{\prime \prime}$ is a the results of a sensitivity analysis which estimates costs based on charges to the NHS, based on the both the Scottish and English reimbursement rates.

Additional file 6. "CONSORT flow diagram of participant journey through trial" illustrates the number of children screened, randomised, and included in the final analysis. This image is taken directly from Maguire et al. 2019.

\section{Abbreviations}

$\mathrm{B}+\mathrm{P}$ : Biological with best practice prevention; $\mathrm{C}+\mathrm{P}$ : Conventional with best practice prevention; CHEERS: Consolidated Health Economic Evaluation Reporting Standards; CONSORT: Consolidated Standards of Reporting Trial; CRF: Case Report Form; FiCTION: Filling Children's Teeth: Indicated or Not?; GA: General Anesthetics; HT: Hall Technique; HTA: Health Technology Assessment; ICER: Incremental cost-effectiveness ratio; IQR: Interquartile Range; ITT: Intention-to-treat; NB: Net Benefit; NHS: National Health Service; NIHR: National Institute of Health Research; PA: Best practice Prevention Alone; SD: Standard Deviation; SUR: Seemingly unrelated regression; UK: United Kingdom

\section{Acknowledgements \\ We thank the Trial Steering Committee: Professor June Nunn (Trinity College, Dublin, Chair), Professor Lorna McPherson (Glasgow), Dr. Ronnie Levine (Leeds), Tina McGuff (Patient representative) and Nicole Douglas (Patient representative); Independent Data Monitoring and Ethics Committee: Professor Helen Worthington (Manchester University, Chair), Professor Marie- Therese Hosey (London) and Professor Gerry Humphris (St Andrews); children and their families who took part in the study, recruiting practices, non-author contributors and the local Clinical Research Networks who supported the trial.}

Authors' contributions

Trial conception: GD, NI, JC, EM. Trial design: AM, GD, NI, JC, EM. Trial conduct: AM, GD, NI, JC, EM, LV. Data acquisition: AM, GD, NI, MR. Data analysis: TH, LV. Data interpretation: TH, AM, GD, NI, JC, NW, VR, EM, MR, LV. 
Drafted manuscript: TH, LV. Critically revised the manuscript: all authors. All authors have read and approved the manuscript.

\section{Funding}

This study was funded by the National Institute for Health Research (NIHR) Health Technology Assessment (HTA) program (project number 07/44/03). The funder was not involved in the design of the study and collection, analysis, and interpretation of data, or in writing the manuscript. The views and opinions expressed therein are those of the authors and not necessarily those of the HTA, NIHR, NHS, or the Department of Health.

\section{Availability of data and materials}

The datasets generated and analysed during the current study are not publicly available due to Regional Ethical Review Board regulations. Any reasonable requests for data should be sent to the corresponding author who, alongside the Trial Management Group, will review the request and give permissions.

\section{Ethics approval and consent to participate}

This study was approved by East of Scotland Research Ethics Committee on 30th July 2012 (REC reference: 12/ES/0047). Parental written consent was obtained and oral or written assent from each child.

\section{Consent for publication}

Not applicable.

\section{Competing interests}

The authors declare no potential conflicts of interest with respect to the conduct of the trial, authorship and/or publication of this article.

\section{Author details}

${ }^{1}$ Population Health Sciences Institute, Newcastle University, Newcastle upon Tyne NE2 4AX, UK. ${ }^{2}$ School of Dental Sciences, Faculty of Medical Sciences, Newcastle University, Newcastle upon Tyne, UK. ${ }^{3}$ Dental School, University of Leeds, Leeds, UK. ${ }^{4}$ School of Dentistry, University of Dundee, Dundee, UK. ${ }^{5}$ Dental Health Services Research Unit, University of Dundee, Dundee, UK.

Received: 25 September 2019 Accepted: 23 January 2020

Published online: 10 February 2020

\section{References}

1. Vernazza CR, Rolland SL, Chadwick B, Pitts N. Caries experience, the caries burden and associated factors in children in England, Wales and Northern Ireland 2013. Br Dent J. 2016;221:315.

2. Scottish Dental. Statement of Dental Remuneration Amendment No. 135. 2017. www.scottishdental.org/wp-content/uploads/2017/09/AmendmentNo.-135-SDR.pdf. Accessed 16 Oct 2017.

3. Public Health Wales. Child Dental General Anaesthetics in Wales. Public Health Wales. 2014.

4. Royal College of Surgeons Faculty of Dental Surgery. The State of Children's Oral Health in England. Royal College of Surgeons. 2015.

5. England NHS. Improving dental care and Oral health - a call to action. NHS England: England; 2014.

6. Ricketts D, Lamont T, Innes NP, Kidd E, Clarkson JE. Operative caries management in adults and children. Cochrane Database Syst Rev. 2013;(3): CD003808.

7. Ahovuo-Saloranta A, Hiiri A, Nordblad A, Worthington H, Makela M. Pit and fissure sealants for preventing dental decay in the permanent teeth of children and adolescents. Cochrane Database Syst Rev. 2017:(7):CD001830.

8. Kidd EA, Fejerskov O. What constitutes dental caries? Histopathology of carious enamel and dentin related to the action of cariogenic biofilms. J Dent Res. 2004;83:Spec No C:C35-8.

9. Marinho VC, Higgins JP, Sheiham A, Logan S. Fluoride toothpastes for preventing dental caries in children and adolescents. Cochrane Database Syst Rev. 2003;(1):CD002278.

10. Drummond MF, Sculpher MJ, Torrance GW, O'Brien BJ, Stoddart GL. Methods for the Economic Evaluation of Health Care Programmes. 3rd ed. New York: Oxford University Press; 2005.

11. Schwendicke F, Krois J, Robertson M, Splieth C, Santamaria R, Innes N. Costeffectiveness of the hall technique in a randomized trial. J Dent Res. 2019; 98(1):61-7.
12. Schwendicke F, Krois J, Splieth CH, Innes N, Robertson M, Schmoeckel J, et al. Cost-effectiveness of managing cavitated primary molar caries lesions: a randomized trial in Germany. J Dent. 2018;78:40-5.

13. Maguire A, Clarkson JE, Douglas GVA, Ryan V, Homer T, Marshman Z, et al. Best-practice prevention alone or with conventional or biological caries management for 3- to 7-year-olds: the FiCTION three-arm RCT. Health Technol Assess. 2019;24(1):1-174.

14. Fayle SA, Welbury RR, Roberts JF. British Society of Paediatric Dentistry: a policy document on management of caries in the primary dentition. Int J Paediatr Dent. 2001;11:153-7.

15. Kindelan SA, Day P, Nichol R, Willmott N, Fayle SA. UK National Clinical Guidelines in Paediatric dentistry: stainless steel preformed crowns for primary molars. Int J Paediatr Dent. 2008;18(Suppl 1):20-8.

16. Innes N, Clarkson J, Speed C, Douglas G, Maguire A. Collaboration. FT. The FiCTION dental trial protocol - filling children's teeth: indicated or not? BMC Oral Health. 2013;13(1):25.

17. Innes NP, Clarkson JE, Douglas GVA, Ryan V, Wilson N, Homer T, et al. Child caries management: a randomized controlled trial in dental practice. J Dent Res. 2019:0(0):0022034519888882.

18. Husereau D, Drummond M, Petrou S, Carswell C, Moher D, Greenberg D, et al. Consolidated health economic evaluation reporting standards (CHEERS) statement. BMJ : Br Med J. 2013;346:f1049.

19. National Institute for Health and Care Excellence (NICE). Guide to the methods of technology appraisal, vol. 2013. London: National Institute for Health and Care Excellence; 2013.

20. Sullivan SD, Mauskopf JA, Augustovski F, Jaime Caro J, Lee KM, Minchin M, et al. Budget impact analysis-principles of good practice: report of the ISPOR 2012 budget impact analysis good practice II task force. Value Health. 2014;17(1):5-14.

21. StataCorp. Stata Statistical Software: Release 14. TX: College Station; 2015. StataCorp LP

22. Fiebig DG. Seemingly unrelated regression. In: Baltagi BH, editor. A companion to theoretical econometrics. Malden, MA, USA: Blackwell Publishing Ltd.; 2003. p. 101-21.

23. Willan AR, Briggs AH, Hoch JS. Regression methods for covariate adjustment and subgroup analysis for non-censored cost-effectiveness data. Health Econ. 2004;13(5):461-75.

24. Barber JA, Thompson SG. Analysis of cost data in randomized trials: an application of the non-parametric bootstrap. Stat Med. 2000;19(23):3219-36.

25. O'Neill C, Worthington HV, Donaldson M, Birch S, Noble S, Killough S, et al. Cost-effectiveness of caries prevention in practice: a randomized controlled trial. J Dent Res. 2017:96(8):875-80.

26. Fenwick E, Claxton K, Sculpher M. Representing uncertainty: the role of costeffectiveness acceptability curves. Health Econ. 2001;10(8):779-87.

27. Hu Y-H, Tsai A, Ou-Yang L-W, Chuang L-C, Chang P-C. Postoperative dental morbidity in children following dental treatment under general anesthesia. BMC Oral Health. 2018;18(1):84

28. Escanilla-Casal A, Ausucua-Ibáñez M, Aznar-Gómez M, Viaño-García JM, Sentís-Vilalta J, Rivera-Baró A. Comparative study of postoperative morbidity in dental treatment under general anesthesia in pediatric patients with and without an underlying disease. Int J Paediatr Dent. 2016;26(2):141-8.

29. American Academy of Pediatric Dentistry. Policy on early childhood caries (ECC): Classifications, consequences, and preventive strategies. Pediatr Dent. 2014;37:15-6.

30. Vermaire $\mathrm{JH}$, van Loveren $\mathrm{C}$, Brouwer WB, Krol M. Value for money: economic evaluation of two different caries prevention programmes compared with standard care in a randomized controlled trial. Caries Res. 2014;48(3):244-53.

31. Samnaliev M, Wijeratne R, Kwon EG, Ohiomoba H, Ng MW. Costeffectiveness of a disease management program for early childhood caries. J Public Health Dent. 2015;75(1):24-33.

32. Lord J, Longworth L, Singh J, Onyimadu O, Fricke J, Bayliss S, et al. Economic analysis of Oral health promotion approaches for dental teams: NICE public health guideline on Oral health promotion approaches for dental teams (GID-PHG60). 2015.

33. Curtis L, Burns A. Unit costs of health and social care. Personal Social Services Research Unit. Canterbury: University of Kent; 2018.

\section{Publisher's Note}

Springer Nature remains neutral with regard to jurisdictional claims in published maps and institutional affiliations. 